\title{
Editoriali
}

\section{The present and future of mental health care in Japan}

\author{
HIROTO ITO
}

\section{INTRODUCTION}

Although the shift from hospital-based psychiatric care to community care began in 1960's, Japan remains the only country in the developed world where deinstitutionalization has yet to significantly occur. As a consequence, elderly and chronic patients who have been living in Japanese psychiatric hospitals for a long time are the typical psychiatric inpatients.

Japan has a universal health care insurance system that mental health care should be available and affordable, at least at a minimum level, to all citizens. Recently, however, the health and social security system has been eroded by a stagnant economy and the medical and social demands of an aging population. At the beginning of the 21 st century, Japan will have the largest aging population that the world has ever witnessed. It is estimated that by 2010 one-third of the entire household members will be 65 years of age or older (Japan Ministry of Health and Welfare, 1997). The more elderly the population of a society, the higher prevalence of dementia they will have. By 2000 , it is estimated that those elderly with dementia will number 1.55 million (Japan Ministry of Health and Welfare, 1999). Where the mentally ill can be cared for, outside of hospitals, is of critical policy concern in Japan.

Unlike in the United States, but similar to many western countries, the Japanese government plays a substantial role in administering health care. But govern-

\footnotetext{
Indirizzo per la corrispondenza: $\mathrm{H}$. Ito, Department of Health Care Economics, National Institute of Health Services Management, 123-1 Toyama, Shinjuku-ku, Tokyo 162-0052, Japan.

Fax: +81-3-3202-6853

E-mail: ito@nih.go.jp
}

mental reform of mental health care is still in its initial stages because of limited understanding of psychiatric illnesses and pervasive stigma. Psychiatric disorders have been greatly underestimated as a cause of disability. Although depression is the leading cause of global morbidity in the world (Murray \& Lopez, 1996), mental diseases are not commonly fatal, and their heavy social burden hides under other more recognized, acceptable and often fatal illnesses such as cancer and heart disease. In minds of many people, stigma is very great about mental illness in Japan. The general public show overwhelmingly negative attitudes and concerns about the risk to society of psychiatric patients, which has dealt an additional blow to mental health policy development and has kept clinical reform at a snail's pace.

Thornicroft \& Tansella (1999) proposed a conceptual framework, which they called the matrix model, to clarify critical issues in developing mental health services. Using two dimensions, the geographical and the temporal, they constructed a $3 \times 3$ matrix. The geographical levels are (1) country/regional, (2) local and (3) patient. The temporal levels are (a) inputs, (b) processes and (c) outcomes. This article describes, according to the matrix model, the present status of Japanese mental health care services and anticipated directions for the future, including the recent revision of the Mental Health Act.

\section{MENTAL HEALTH CARE SERVICES}

Under the Mental Health Act, patients with mental illness are defined as persons with schizophrenia, acute or dependent substance use disorders, intellectual disability (mental retardation), and other mental conditions 
or diseases, which are in ICD-10 (Seishin Hoken Fukushi Kenkyu-kai, 1999). Estimates are that 2.17 million people in Japan are suffering from metal illness; of these, 336,475 are inpatients, 97,780 are 65 years old or over, and 36,554 are demented (Japan Ministry of Health and Welfare, 1999).

Psychiatric care is provided mainly in three different settings: psychiatric hospitals, general hospitals and clinics. When there are 20 or more inpatient beds, an institution is called a «hospital», and in cases of fewer than 20 beds, it is called a «clinic». Psychiatric clinics in Japan, however, have no beds. There are 263,985 psychiatric beds in 1,057 psychiatric hospitals, 96,911 psychiatric beds in 611 general hospitals (figure 1); and no beds in any of the existing 3,198 psychiatric clinics (Japan Ministry of Health and Welfare, 1996). General hospitals, in this context, mean hospitals other than psychiatric hospitals, and include university hospitals and national medical centers. Generally, chronic long-stay patients are predominant in psychiatric hospitals while patients in acute phases if illness is treated in general hospitals.

In 1999, the number of registered psychiatrists by the Ministry of Health and Welfare was 10,161 (Seishin Hoken Fukushi Kenkyu-kai, 1999). There are psychiatrists who are not registered. The Japanese Society of Psychiatry and Neurology does not have a certification system for psychiatrists; therefore, any medical doctor can become a psychiatrist after passing a national examination. Specific clinical experience and training are required to be a registered psychiatrist. Registered psychiatrists assess dangerousness to self and others for involuntary hospital admission.

Other psychiatric staff include nurses, psychiatric social workers (PSW), clinical psychologists and occupational therapists. Although clinical psychologists in the United States can have their own patients and provide psychotherapy, their scope of practice in Japan is very limited. They mainly perform psychological tests and rarely provide psychotherapy. More strict qualifications are beginning to be discussed. Since 1997, a national examination has been required for psychiatric social workers. Clinical psychologists have to be certified by the psychological association.

The most common ICD-10 diagnoses among inpatients are schizophrenia (64.9\%), dementia (10.9\%), and mood disorders (6.7\%); among outpatients, the diagnoses are schizophrenia $(26.7 \%)$, neuroses $(24.3 \%)$, and mood disorders $(21.3 \%)$ (Ito \& Sederer, 1999). Inpatient length of stay in Japan for the mentally ill is gradually shortening but it is still significantly higher than in other countries. The average length of stay for discharged psychiatric patients in 1993 was 333.3 days in Japan, 18.5 days in Italy, 10.3 days in United States, 46.7 days in Germany, and 86.4 days in United Kingdom (1993: Organization for Economic Development and Cooperation, 1998). The length of stay for inpatients and discharged patients in Japan is even longer; 423.7 days in 1997 (Ito \& Sederer, 1999). In Japan, acute, chronic and forensic patients

Number of Psychiatric Beds

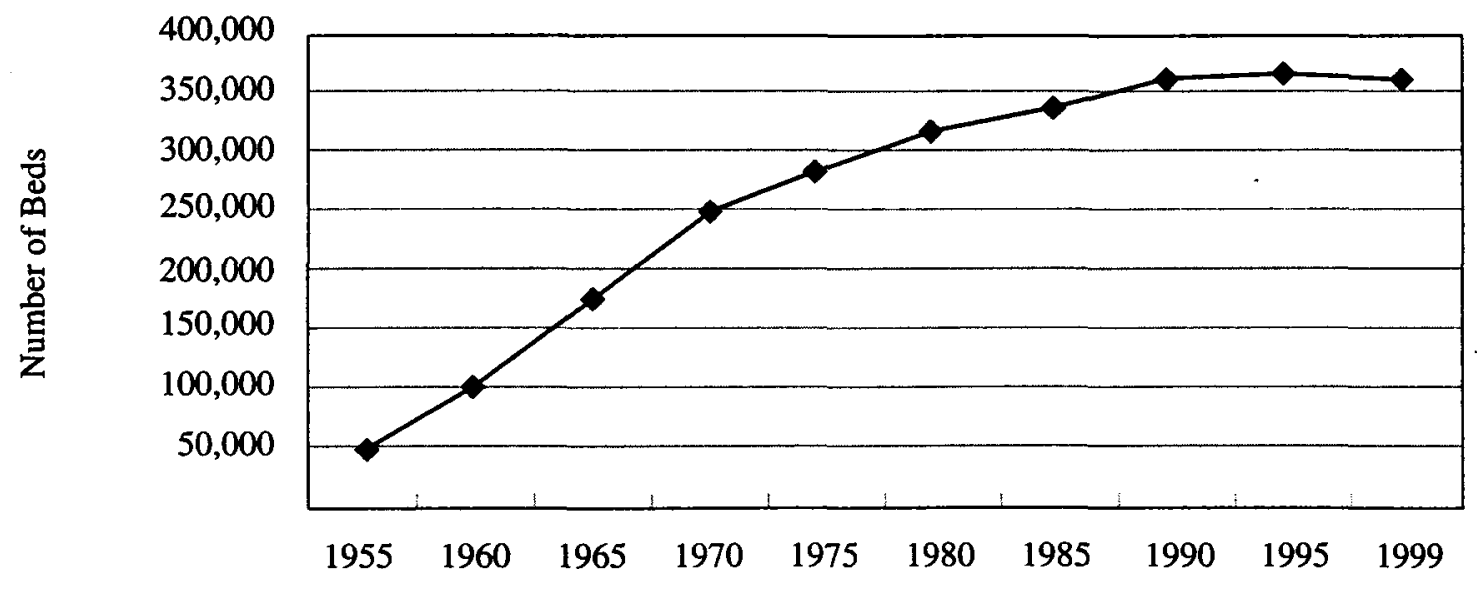

Figure 1. - Number of psychiatric beds (1955-1999).

Year

Epidemiologia e Psichiatria Sociale, 9, 2, 2000 
Table I. - Types of admission.

\begin{tabular}{|c|c|c|}
\hline & June 30, 1998 & June 30,1988 \\
\hline Involuntary Admission for dangerousness & $4,246(1.3 \%)$ & $18,353(5.3 \%)$ \\
\hline Involuntary Admission under guardianship & $91,348(27.5 \%)$ & $276,959(80.3 \%)$ \\
\hline Voluntary Admission & $231,004(69.6 \%)$ & $0 \quad(0 \%)$ \\
\hline Other & $5,272(1.6 \%)$ & $49,485(14.4 \%)$ \\
\hline Total & $331,870(100 \%)$ & $344,797(100 \%)$ \\
\hline
\end{tabular}

Seishin Hoken Kenkyu-kai (1999) and Japan Ministry of Health and Welfare (1999)

Table II. - Number of rehabilitation facilities.

19952002

Estimated under the present pian

\begin{tabular}{llcc}
\hline Rehabilitation & & 248 & 1,059 \\
& Number & 4220 & 19,770 \\
Group home & Capacity & 220 & 920 \\
& Number & 1,210 & 5,060 \\
Daycare & Resident & 372 & 1,000 \\
& Number & 18,600 & 50,000 \\
\hline
\end{tabular}

Seishin Hoken Kenkyu-kai (1999)

are mixed together in the same psychiatric units and, as the long length of stay suggests, most of them receive custodial and chronic care.

Admissions to hospitals may be involuntary or voluntary. There are two types of involuntary admissions in Japan: (1) compulsory admission by order of the prefectural (similar to «state» in the United States) governor for a patient who is dangerous to self or others and (2) compulsory admission under guardianship for a patients who is not dangerous to self or others but does not consent to admission (Seishin Hoken Fukushi Kenkyukai, 1999). For the second type of admission, the patient is hospitalized when there is consent of a guardian, usually a family member, and an order by a psychiatrist. Until recently, guardian admissions were the most common type in Japan. With recent efforts to protect patient rights, voluntary admissions increased from $0 \%$ in 1988 to $69.6 \%$ in 1998 (table I).

\section{DIRECTIONS FOR THE FUTURE}

Since consensus is highly valued in Japan, new policies feature slow and gradual change. However, new directions are emerging for the future of mental health care in Japan. The principal issues and challenges are described below, according to the matrix model, by focusing on aspects of national, local and patient levels, and inputs, processes and outcomes.

\section{National Level}

Since Japan has a form of national health service, there is highly centralized control. National initiatives include differentiation of care by specialty services (which is being attempted by the force of new law) and changes in the management of reimbursement (which is discussed in «inputs phase»). Adult long-stay patients are targeted for release to the community. Very old patients are now going to nursing home or dementia facilities, while other long-stay patients are being sent to non-hospital residential facilities such as group homes and supported housing. Some patients will be able to go back to their homes with day care or occupational training services. For achieve the goal of discharge to the community, the government plans to increase residential facilities and social services in the communities (table II). The severe and persistently ill will remain in psychiatric hospitals. 


\section{Local Level}

Prevention efforts of local health care centers, mostly maternal child health and infection control, have greatly contributed to making the Japanese population experience the lowest infant mortality and the longest life span in the world. A negative aspect of the highly centralized control, however, is that there are very few independent local authorities. Particularly, in mental health, each local population is not yet required to undergo evaluation. Decentralization will be necessary to enable deinstitutionalization to succeed; catchmentarea responsibility should be encouraged in mental health. Needs assessments should be conducted to understand differences among geographical regions in the development of alternative facilities for the mentally ill living in the communities.

\section{Patient Level}

"Normalization» of the mentally ill and protection of human rights were strengthened by the revision of the Mental Health Act (Seishin Hoken Fukushi Kenkyu-kai, 1999). The law provides the right to live, work and participate in the community, without negative discrimination. In Japan, the family is central to social support; the role of the family in mental health care is very prominent. Familyís supervision has been required by law, but recent aging of family caregivers prompted a reduction in the requirements of families for caretaking in the 1999 revision of the Mental Health Act.

\section{Input Phase}

Not only psychiatric care but also other health care services have been managed by the government of Japan using mostly structural aspects of services, for example, staffing, size of rooms and units, so-called «visible inputs» in a matrix model. Reimbursement has been determined by a structural «facility standard». Thus, almost all attention to service design has given to structural aspects by the government, the Japan Medical Association and the hospital associations.

In order to achieve differentiation of care into acute and chronic care, what changes in the number of psychiatrists and other staff members (staff to patient ratios) and the size of rooms and units are required is being studied. By law and regulation, psychiatric beds now require only one third the number of physicians required for other medical care units. Acute care units, where intensive care is provided, will require greater numbers of psychiatrists.

Inpatient payment for beds on a psychiatric unit was one-third less than that of general medical units, which had the effect of an incentive for more beds and longer stays. The maximum number of beds, however, was regulated by the 1990 revision of the Medical Care Act; consequently, most psychiatric hospitals no longer increase number of their beds. A recent policy step by the government has been to promote reduced lengths of stay by a new reimbursement system. The same reimbursement as exists for general medical units is now available for acute psychiatric units if $40 \%$ of patients are discharged to their home or to residential facilities for the mentally ill within three months (Ito \& Sederer, 1999). This financial incentive seems to be working as evidenced by recently hospitalized patients tending to be discharged with shorter lengths of stay than longstay patients.

\section{Process Phase}

The process phase refers to clinically related procedures in the delivery of care (i.e., what is done for and to the patient and the clinical care). Processes include management of medical chart, clinical guidelines and protocols, and monitoring of clinical care, including high risk events. In Japan, seclusion and restraint of inpatients is one of the most focused process issues today.

Japanese psychiatric hospitals were developed from clinics where mostly one psychiatrist provided outpatient care to patients. This historical background of hospital management has meant that hospitals tend to manage operational processes more as an organization than a hospital. Borrowing from a British model of care, the Japanese government established a Psychiatric Review Board to monitor mental health services in 1988 (Japan Ministry of Health and Welfare, 1999). Although inpatients could appeal to the Psychiatric Review Board at any time by a telephone call, its use has been very low. The 1999 revision of the Mental Health Act, however, is attempting to strengthen the functions of the Psychiatric Review Board (Seishin Hoken Fukushi Kenkyu-kai, 1999).

The Japan Association of Psychiatric Hospitals began "peer review» inspections in 1993. A counterpart of Joint Commission on the Accreditation of Healthcare Organizations (JCAHO) in the United States, the Council for Quality Health Care (JCQHC), was established in 1995 and began to conduct the third-party evaluations of health 
care organizations in 1997 (Ito et al., 1998). These activities focus especially on process evaluation.

\section{Outcome Phase}

Outcomes are the results of treatment and care rendered. In Japan, outcomes have received little attention in mental health. Studies on pharmacological efficacy were carried out, but there have been no attempts to evaluate the effectiveness of health care practices: Recently, however, increasing attention has been given to quality of care, as well as cost effectiveness and, for measuring quality of care (Sederer \& Dickey, 1996). Improvements in the functional status and quality of life in patients, readmission rates in relation with shorter length of stay, and patient satisfaction have become important outcome indicators.

The first patient satisfaction survey in Japanese psychiatric hospitals was conducted in 1997. Some interesting results were found in 326 discharged patients from 31 psychiatric hospitals: patients with personality disorder and younger patients were less satisfied than other psychiatric patients, and involuntary patients rated lower satisfaction regarding the provision of informed consent than voluntary patients (Ito et al., 1999).

The development of measurement tools is underway to continuously evaluate improvement of patient symptoms, quality of care and satisfaction. Using these tools and risk adjustment methods, a nationwide outcome survey is planned to be carried out within a few years. Eventually, this will lead to development of a reimbursement model that will be based on outcomes.

\section{TOWARD REFORM}

In Japan, where most psychiatric patients are still in hospitals, many challenges are ahead in the path of mental health reform: lack of residential facilities for patients who could be discharged from hospitals; effective care for the demented and patients with psychiatric and me- dical illnesses (co-morbid conditions); differentiation of specialty care into acute, chronic and forensic care; adequate staffing; a reimbursement system that rewards effective care; continuity of care; and patient rights. These are the goals that mental health reform is gradually moving toward.

With greater accountability in health care in mind, «evidence-based" treatments will be very important for policymakers and health care providers. Improvement in data gathering and analysis will be crucial for future planning of the infrastructure of delivery of mental health care services. Balancing financial concerns with quality of care remains the ultimate political agenda in Japan.

\section{REFERENCES}

Ito H. \& Sederer L.I. (1999). Mental health services reform in Japan. Harvard Review of Psychiatry 7, 208-215.

Ito H., Iwasaki S., NakanoY., Imanaka Y., Kawakita H. \& Gunji, A. (1998). Direction of quality improvement activities of health care organizations in Japan. International Journal for Quality in Health Care 10, 361-363.

Ito H., Shingai N., Yamazumi S., Sawa Y. \& Iwasaki S. (1999). Patient perceptions and satisfaction of psychiatric services at their discharge [in Japanese]. Psychiatria et Neurologia Japonica 101, 138 147.

Japan Ministry of Health and Welfare (1996). 1996 Health Facilities Survey [in Japanese]. Kousei Tokei Kyokai: Tokyo.

Japan Ministry of Health and Welfare (1997). Annual Report on Health and Welfare: 1995-1996. Japan International Corporation of Welfare Services: Tokyo.

Japan Ministry of Health and Welfare (1999). Mental Health and Welfare in Japan: Mental Health and Welfare Handbook [in Japanese]. Kouken Syuppan: Tokyo.

Murray C.J. \& Lopez A. D. (1996). The Global Burden of Disease: A Comprehensive Assessment of Mortality and Disability From Diseases Injuries, and Risk Factors in 1990 and Projected. WHO: Geneva.

Organization for Economic Development and Cooperation (1998). OECD Health Data 98. OECD Health Policy Unit: Paris.

Sederer L.I. \& Dickey B. (1996). Outcomes Assessment in Clinical Practice. Williams and Wilkins: Baltimore.

Seishin Hoken Fukushi Kenkyu-kai (1999). New Mental Health Act [in Japanese]. Cyuo Houki: Tokyo.

Thornicroft G. \& Tansella M. (1999). The Mental Health Matrix: A Manual to Improve Services. Cambridge University Press: Cambridge. 